\title{
Effect of Perceived Social Support on Postnatal Depression in Females with Cesarean Section and Normal Vaginal Delivery
}

\section{Muhammad Asif',*, Muhammad Saleem Rana ${ }^{1}$, Asif Hanif', Syed Amir Gilani', Ume Habiba ${ }^{2}$ Sultan Ayaz ${ }^{3}$, Benish Ali ${ }^{4}$}

1 University Institute of Public Health, The University of Lahore, Pakistan.

${ }^{2}$ Directorate of Medical Sciences, Department of Public Health, Government College University Faisalabad,

Pakistan.

${ }^{3}$ Directorate of Medical Sciences, Department of Eastern Medicine, Government College University Faisalabad, Pakistan.

${ }^{4}$ Directorate of Medical Sciences, Department of Orthotics and Prosthetics, Government College University Faisalabad.

\section{Authors' Contributions \\ 1 Conception \& Study Design, Data Collection \& Processing, Data Analysis and/or Interpretation, Drafting of Manuscript. \\ 2 \& 3 Conception \& Study Design, Critical Review. \\ 4, 6 \& 7 Critical Review. \\ 5 Data Collection \& Processing, Data Analysis and/or Interpretation.}

\section{Acknowledgement}

\section{Article info.}

Received: January 14, 2021

Accepted: February 08, 2021

Funding Source: Nil

Conflict of Interest: Nil

Cite this article: Asif M, Rana MS, Hanif A, Gilani SA, Habiba U, Ayaz S, Ali B. Effect of Perceived Social Support on Postnatal Depression in Females with Cesarean Section and Normal Vaginal Delivery. RADS J Pharm Pharm Sci. 2020; 8(4):222-230.

*Address of Correspondence Author: muhammadasif@gcuf.edu.pk

\begin{abstract}
A B S TR A C T
Objectives: To assess the association of perceived social support with postnatal depression among puerperal women of Faisalabad, Pakistan.

Methods: It was a case-control study carried out during a period of 18 months (1-1-2019 to 30-06-2020). By using purposive sampling technique 284 postpartum women were enrolled from Allied hospital and Children hospital Faisalabad. The postnatal depression was diagnosed with Edinburg Postnatal Depression Scale (EPDS).The Multidimensional Scale of Perceived Social Support (MSPSS) was used to measure the social support that was perceived by women.
\end{abstract}

Results: According to cut-off score $\geq 13$ on EPDS $37.3 \%$ women were found depressed while $62.7 \%$ women were found non-depressed. The mean EPDS score \pm SD was $10.12 \pm 6.27$ ( $\min$ score 0 ; max score 27 ). The perceived social support from significant other, family and friends was found as significant predictor of postnatal depression. With one-point increase in global perceived social support, the postnatal depression decreased by 0.1 points on EPDS scale $(p=0.03)$ among women in normal vaginal delivery group, by 0.2 points in cesarean section group $(p<0.001)$ and the postnatal depression decreased by 0.2 points in total sample $(p<0.001)$.

Conclusion: A poor perceived social support from significant other, family and friends is a significant risk factor for postnatal depression among puerperal women.

Keywords: Postnatal Depression, EPDS, MSPSS, Perceived social support.

\section{INTRODUCTION}

Postpartum or postnatal depression is currently known as a notable health problem because it has many adverse effects not only on the health of the mother but also on the health of the infant [1].In South Asia, depression during childbirth is a serious public health problem, affecting approximately one in four women $[2,3]$.The estimated prevalence rate for this disease nationwide is commonly thought to be about 
$13 \%[4,5]$. Without a question, that rate is concerning, suggesting an immediate need for more in-depth research into the causes and conditions contributing to this disease, since it has detrimental impact on women, children and community as a whole[6].During the first four weeks including the postnatal period, postpartum depression (PPD) occurs which can persist since as long as one year[7].This not only affects wellbeing of the mother, but also has a detrimental impact on the mental, cognitive, psychological and even functional growth of the child[8].

Several risk factors of postnatal depression have been identified, for example, obstetric history, physical causes, behavior, traumatic life events, past mental illness background and socialsupport [9].The risk factors of postnatal depression identified in multivariate analysis done by Roumieh et al, were history of depression for the period of pregnancy, worrying life occasions and displacement, while antenatal care was found as a protective factor for postnatal depression [10]. Smorti et al identified women's age, prenatal depression during pregnancy, quality of relationship with spouse and mode of delivery as significant predictors of postnatal depression [11]. Other risk factors of postnatal depression identified in literature are socioeconomic status, biological history of mother, obstetrical history, lifestyle and previous mental illnesses [12, 13].

Surveys and epidemiological studies report that the incidence of postpartum depression in different cultures around the world is increasing [3]. In order to determine whether this issue is specific to a particular cultural background or is significantly affected by it, it is important to determine the prevalence of PPD and its social and biological relevance in different populations and cultures [14]. This study aimed to assess the association of perceived social support with postnatal depression among puerperal women of Faisalabad, Pakistan.

\section{MATERIALS AND METHODS}

A case-control study design was used in this study. Women diagnosed with postnatal depression were taken as cases and those women who had no depression were enrolled as controls. The exposure to various risk factors was compared between cases and controls. The study was conducted in Allied hospital Faisalabad and Children hospital Faisalabad.
The study was completed in 18 months after approval of synopsis i-e, from 4th Jan 2019 to 5th July 2020.A non-probability A sample of 284 women (142 women in normal delivery group; 142 women in cesarean section group) was taken by using $90 \%$ power of test, $95 \%$ confidence level and 5\% margin of error using following formula:

$\mathrm{n}=\frac{\left\{z_{1-\alpha} \sqrt{2 \overline{\mathrm{P}}(1-\overline{\mathrm{P}})}+z_{1-\beta} \sqrt{\mathrm{P}_{1}\left(1-\mathrm{P}_{1}\right)+\mathrm{P}_{2}\left(1-\mathrm{P}_{2}\right)}\right\}^{2}}{\left(\mathrm{P}_{1}-\mathrm{P}_{2}\right)^{2}}$

$\mathrm{P} 1=$ Depression in females with normal vaginal delivery $=13.6 \%[15]$

$\mathrm{P} 2=$ Depression in females with C-section $=27.6 \%$ [15]

Purposive sampling technique was used to collect data. Postpartum women aged 18-45 years were included in the study. Women who had any kind of condition that required immediate inpatient care, women who had stillbirth, neonatal death or newborn with any kind of physical or mental anomalies, women who had elective cesarean section without any medical recommendation or complication, women who had their newborn less than one month and more than 6 months of age and women who could not speak Urdu or Punjabi language were excluded from the study.

The rules and regulations set by the ethical committee of university of Lahore were followed while conducting the research and the rights of the research participants were respected.

The Edinburg Postnatal Depression Scale (EPDS) was used to measure the prevalence of postnatal depression among women of this study. The Multidimensional Scale of Perceived Social Support (MSPSS) was used to measure the social support that was perceived by women. This scale consists of 12 items that are measured on 7 points Likert scale, from 'very strongly disagree' to 'very strongly agree'. The 12 items of MSPSS are further categorized into three subscales measuring 'perceived social support from significant other', 'perceived social support from family' and 'perceived social support from friends' separately. Each subscale consists of 4 items. The minimum possible score was 4 and maximum possible score was 28. A higher score indicated higher social support perceived by women of this study. The overall score of all 12 items measured 'Global Perceived Social Support'. The minimum possible score for 'Global Perceived Social Support' 
was 7 and maximum possible score was 84 . A higher score indicated higher 'Global Perceived Social Support'.

\section{Data Analysis}

All the data was analyzed by using SPSS version 24 . The women screened out with postnatal depression (EPDS score >13) were compared with women having no depression across perceived social support variables using the Chi-square Test of Independence. A Simple Linear Regression was done to measure the association of 'global perceived social support' with postnatal depression among women in cesarean section group and normal vaginal delivery group separately. A p-value $<0.05$ was considered as showing statistically significant results for all statistical tests used in this study.

\section{RESULTS}

A total of 284 postpartum women (142 having normal vaginal delivery and 142 having cesarean section) were enrolled in the study. According to cut-off score $\geq 13$ on EPDS 106 (37.3\%) women were found depressed while 178 (62.7\%) women were found non-depressed. The mean EPDS score \pm SD was $10.12 \pm 6.27$ (min score 0 ; $\max$ score 27$)$. The descriptive statistics of Perceived social support scale and subscales among women having normal vaginal delivery $(n=142)$ and cesarean section $(n=142)$ are shown below in Table 1.

Table 1. Descriptive Statistics of Perceived Social Support Scale and Subscalesamong Women Having Normal Vaginal Delivery $(n=142)$ and Cesarean Section $(n=142)$.

\begin{tabular}{|c|c|c|c|c|c|}
\hline Scale/Subscale & Study Groups & $\mathbf{N}$ & Min & Max & Mean \pm SD \\
\hline $\begin{array}{c}\text { Perceived Social Support from } \\
\text { Significant Other } \\
\text { (Subscale) }\end{array}$ & Normal Vaginal Delivery & 142 & 9 & 28 & $21.50 \pm 4.59$ \\
\cline { 2 - 5 } & Cesarean Section & 142 & 5 & 28 & $20.23 \pm 5.57$ \\
\cline { 2 - 5 } & Total & 284 & 5 & 28 & $20.87 \pm 5.14$ \\
\hline $\begin{array}{c}\text { Perceived Social Support from } \\
\text { Family } \\
\text { (Subscale) }\end{array}$ & Normal Vaginal Delivery & 142 & 12 & 28 & $21.09 \pm 4.39$ \\
\cline { 2 - 5 } & Cesarean Section & 142 & 4 & 28 & $20.12 \pm 5.34$ \\
\cline { 2 - 5 } $\begin{array}{c}\text { Sorceived Social Support from } \\
\text { Friends } \\
\text { (Subscale) }\end{array}$ & Total & 284 & 4 & 28 & $20.61 \pm 4.91$ \\
\cline { 2 - 5 } & Cesmal Vaginal Delivery & 142 & 12 & 28 & $20.22 \pm 3.58$ \\
\cline { 2 - 5 } & Total & 284 & 8 & 28 & $19.86 \pm 4.51$ \\
\hline $\begin{array}{c}\text { Global Pection } \\
\text { Support } \\
\text { (Scale) }\end{array}$ & Normal Vaginal Delivery & 142 & 36 & 84 & $62.82 \pm 10.99$ \\
\cline { 2 - 5 } & Cesarean Section & 142 & 19 & 84 & $59.85 \pm 14.95$ \\
\cline { 2 - 5 } & Total & 284 & 19 & 84 & $61.34 \pm 13.18$ \\
\hline
\end{tabular}

The Chi-square Test of Independencerevealed that the women's perception that they have someone special who is there in time of need was not significantly associated with postnatal depression among women in normal vaginal delivery group $x^{2}(2$, $\mathrm{n}=142)=3.09, \mathrm{p}=0.21$; but was significantly associated with postnatal depression among women in cesarean section group $X^{2}(2, n=142)=17.05, p<$ 0.001 ; and in total sample $X^{2}(2, n=284)=16.79, p<$ 0.001 . The women's perception that they have someone special to share worries and happiness with $\mathrm{him} / \mathrm{her}$ was significantly associated with postnatal depression among women in normal vaginal delivery group $\mathrm{X}^{2}(2, \mathrm{n}=142)=9.77, \mathrm{p}=0.008$; among women in cesarean section group $X^{2}(2, n=142)=$ $21.40, p<0.001$; as well as in total sample $X^{2}(2, n=$ $284)=34.92, p<0.001$. The women's perception that they have someone special who provides real comfort to them was significantly associated with postnatal depression among women in normal vaginal delivery group $x^{2}(2, n=142)=14.13, p=0.001$; among women in cesarean section group $\chi^{2}(2, n=142)=$ $20.74, p<0.001$; as well as in total sample $X^{2}(2, n=$ $284)=31.88, p<0.001$. The women's perception that they have someone special for whom their feelings matter a lot was significantly associated with postnatal depression among women in normal vaginal delivery group $x^{2}(2, n=142)=7.56, p=0.02$; among 
women in cesarean section group $x^{2}(2, n=142)=$ $13.70, p<0.001$; as well as in total sample $X^{2}(2, n=$ 284) $=23.95, p<0.001$ (Table 2).

The Chi-square Test of Independencerevealed that the women's perception that they have a helping family was significantly associated with postnatal depression among women in normal vaginal delivery group $\mathrm{X}^{2}(2, \mathrm{n}=142)=18.37, \mathrm{p}<0.001$; among women in cesarean section group $x^{2}(2, n=142)=$ $46.21, p<0.001$; as well as in total sample $x^{2}(2, n=$ $284)=68.86, p<0.001$. The women's perception that they are emotionally supported and being helped by family was significantly associated with postnatal depression among women in normal vaginal delivery group $\mathrm{X}^{2}(2, \mathrm{n}=142)=19.61, \mathrm{p}<0.001$; among women in cesarean section group $X^{2}(2, n=142)=$ $30.39, p<0.001$; as well as in total sample $x^{2}(2, n=$ $284)=50.23, p<0.001$. The women's perception that they can consult their family in case of any problem was significantly associated with postnatal depression among women in normal vaginal delivery group $X 2(2$, $\mathrm{n}=142)=9.14, \mathrm{p}=0.01$; among women in cesarean section group $x^{2}(2, n=142)=19.38, p<0.001$; as well as in total sample $X^{2}(2, n=284)=25.43, p<$ 0.001 . The women's perception that their family helps in decision making matters was significantly associated with postnatal depression among women in normal vaginal delivery group $x 2(2, n=142)=$ 24.26, $\mathrm{p}<0.001$; among women in cesarean section group $X^{2}(2, n=142)=34.57, p<0.001$; as well as in total sample $X^{2}(2, n=284)=62.21, p<0.001$ (Table 2).

The Chi-square Test of Independencerevealed that the women's perception that their they have some helping friends was significantly associated with postnatal depression among women in normal vaginal delivery group $x^{2}(2, n=142)=21.02, p<0.001$; among women in cesarean section group $x^{2}(2, n=$ $142)=21.83, p<0.001$; as well as in total sample $x^{2}$ $(2, \mathrm{n}=284)=41.79, \mathrm{p}<0.001$. The women's perception that they are able to rely on friends when matters go wrong was not significantly associated with postnatal depression among women in normal vaginal delivery group $x^{2}(2, n=142)=0.72, p=0.69$; among women in cesarean section group $X^{2}(2, n=$ $142)=2.02, p=0.36$; as well as in total sample $x^{2}(2$, $\mathrm{n}=284)=2.38, \mathrm{p}=0.30$. The women's perception that they have friends to share worries and happiness with them was significantly associated with postnatal depression among women in normal vaginal delivery group $X^{2}(2, n=142)=7.80, p=0.02$; among women in cesarean section group $X^{2}(2, n=142)=10.82, p=$ 0.004 ; as well as in total sample $x^{2}(2, n=284)=$ 12.39, $p=0.002$. The women's perception that they can consult their friends in case of any problem was significantly associated with postnatal depression among women in normal vaginal delivery group $X^{2}(2$, $\mathrm{n}=142)=11.80, \mathrm{p}=0.003$; among women in cesarean section group $x^{2}(2, n=142)=7.28, p=$ 0.02 ; as well as in total sample $x^{2}(2, n=284)=$ 17.50, $\mathrm{p}<0.001$ (Table 2).

Table 2. Chi-Square Test of Independence to Measure Association of Perceived Social Support from Significant other with Postnatal Depression Among Women Having Normal Vaginal Delivery $(n=142)$ and Cesarean Section $(n=142)$.

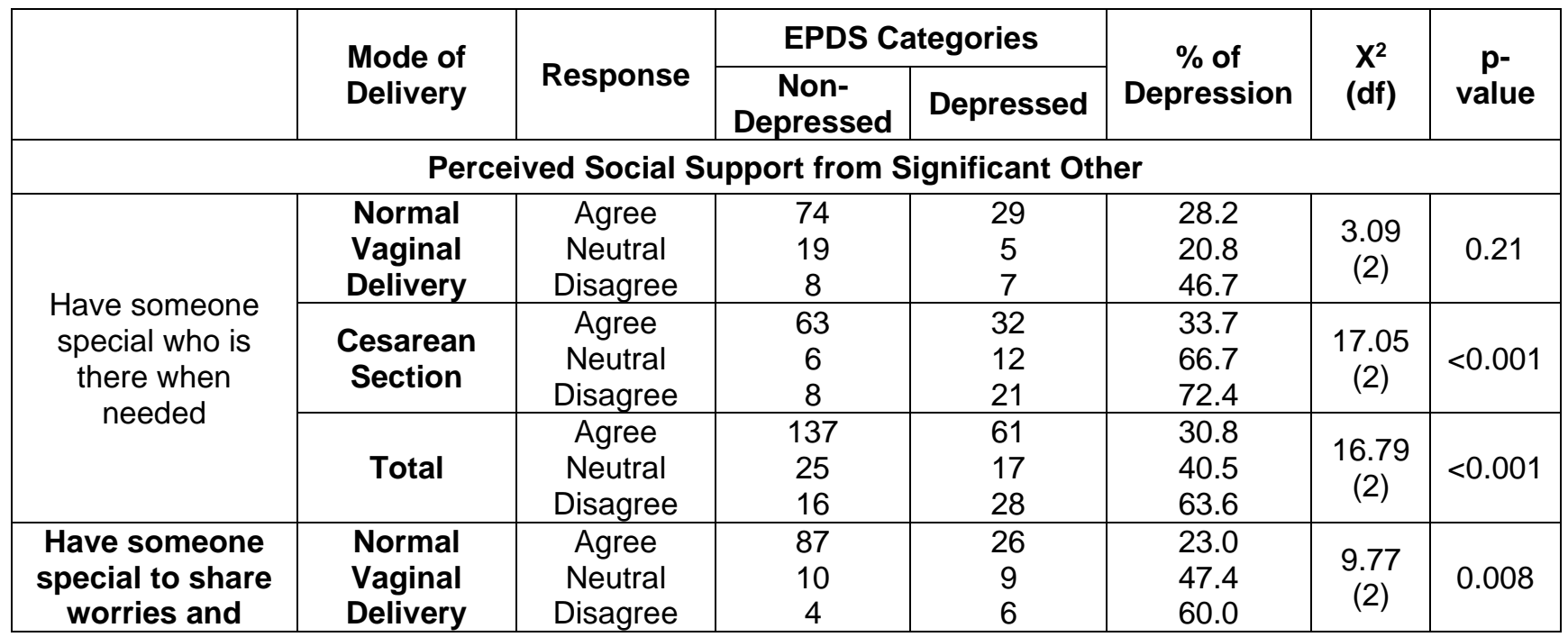




\begin{tabular}{|c|c|c|c|c|c|c|c|}
\hline \multirow[t]{2}{*}{$\begin{array}{l}\text { happiness with } \\
\text { him/her }\end{array}$} & $\begin{array}{l}\text { Cesarean } \\
\text { Section }\end{array}$ & $\begin{array}{c}\text { Agree } \\
\text { Neutral } \\
\text { Disagree }\end{array}$ & $\begin{array}{c}65 \\
8 \\
4\end{array}$ & $\begin{array}{l}32 \\
15 \\
18\end{array}$ & $\begin{array}{l}33.0 \\
65.2 \\
81.8\end{array}$ & $\begin{array}{c}21.40 \\
(2)\end{array}$ & $<0.001$ \\
\hline & Total & $\begin{array}{c}\text { Agree } \\
\text { Neutral } \\
\text { Disagree }\end{array}$ & $\begin{array}{c}152 \\
18 \\
8\end{array}$ & $\begin{array}{l}58 \\
24 \\
24\end{array}$ & $\begin{array}{l}27.6 \\
57.1 \\
75.0\end{array}$ & $\begin{array}{c}34.92 \\
(2)\end{array}$ & $<0.001$ \\
\hline \multirow{3}{*}{$\begin{array}{l}\text { Got someone } \\
\text { special who } \\
\text { provides real } \\
\text { comfort }\end{array}$} & $\begin{array}{l}\text { Normal } \\
\text { Vaginal } \\
\text { Delivery }\end{array}$ & $\begin{array}{c}\text { Agree } \\
\text { Neutral } \\
\text { Disagree }\end{array}$ & $\begin{array}{c}81 \\
14 \\
6\end{array}$ & $\begin{array}{l}21 \\
10 \\
10\end{array}$ & $\begin{array}{l}20.6 \\
41.7 \\
62.5\end{array}$ & $\begin{array}{c}14.13 \\
(2)\end{array}$ & 0.001 \\
\hline & $\begin{array}{c}\text { Cesarean } \\
\text { Section }\end{array}$ & $\begin{array}{l}\text { Agree } \\
\text { Neutral } \\
\text { Disagree }\end{array}$ & $\begin{array}{c}62 \\
3 \\
12 \\
\end{array}$ & $\begin{array}{l}30 \\
16 \\
19 \\
\end{array}$ & $\begin{array}{l}32.6 \\
84.2 \\
61.3 \\
\end{array}$ & $\begin{array}{l}20.74 \\
(2)\end{array}$ & $<0.001$ \\
\hline & Total & $\begin{array}{c}\text { Agree } \\
\text { Neutral } \\
\text { Disagree }\end{array}$ & $\begin{array}{c}143 \\
17 \\
18\end{array}$ & $\begin{array}{l}51 \\
26 \\
29\end{array}$ & $\begin{array}{l}26.3 \\
60.5 \\
61.7 \\
\end{array}$ & $\begin{array}{c}31.88 \\
(2)\end{array}$ & $<0.001$ \\
\hline \multirow{3}{*}{$\begin{array}{l}\text { Have someone } \\
\text { special for } \\
\text { whom my } \\
\text { feelings matter a } \\
\text { lot }\end{array}$} & $\begin{array}{l}\text { Normal } \\
\text { Vaginal } \\
\text { Delivery }\end{array}$ & $\begin{array}{c}\text { Agree } \\
\text { Neutral } \\
\text { Disagree }\end{array}$ & $\begin{array}{c}80 \\
17 \\
4\end{array}$ & $\begin{array}{c}26 \\
8 \\
7\end{array}$ & $\begin{array}{l}24.5 \\
32.0 \\
63.6\end{array}$ & $\begin{array}{c}7.56 \\
(2)\end{array}$ & 0.02 \\
\hline & $\begin{array}{c}\text { Cesarean } \\
\text { Section }\end{array}$ & $\begin{array}{c}\text { Agree } \\
\text { Neutral } \\
\text { Disagree }\end{array}$ & $\begin{array}{c}62 \\
11 \\
4\end{array}$ & $\begin{array}{c}39 \\
8 \\
18\end{array}$ & $\begin{array}{l}38.6 \\
42.1 \\
81.8 \\
\end{array}$ & $\begin{array}{c}13.70 \\
(2)\end{array}$ & 0.001 \\
\hline & Total & $\begin{array}{c}\text { Agree } \\
\text { Neutral } \\
\text { Disagree }\end{array}$ & $\begin{array}{c}142 \\
28 \\
8\end{array}$ & $\begin{array}{l}65 \\
16 \\
25\end{array}$ & $\begin{array}{l}31.4 \\
36.4 \\
75.8\end{array}$ & $\begin{array}{c}23.95 \\
(2)\end{array}$ & $<0.001$ \\
\hline \multicolumn{8}{|c|}{ Perceived Social Support from Family } \\
\hline \multirow{3}{*}{$\begin{array}{l}\text { Have a helping } \\
\text { family }\end{array}$} & $\begin{array}{l}\text { Normal } \\
\text { Vaginal } \\
\text { Delivery }\end{array}$ & $\begin{array}{c}\text { Agree } \\
\text { Neutral } \\
\text { Disagree }\end{array}$ & $\begin{array}{c}89 \\
4 \\
8\end{array}$ & $\begin{array}{c}23 \\
8 \\
10\end{array}$ & $\begin{array}{l}20.5 \\
66.7 \\
55.6\end{array}$ & $\begin{array}{l}18.37 \\
(2)\end{array}$ & $<0.001$ \\
\hline & $\begin{array}{c}\text { Cesarean } \\
\text { Section }\end{array}$ & $\begin{array}{c}\text { Agree } \\
\text { Neutral } \\
\text { Disagree }\end{array}$ & $\begin{array}{c}70 \\
3 \\
4\end{array}$ & $\begin{array}{l}24 \\
24 \\
17\end{array}$ & $\begin{array}{l}25.5 \\
88.9 \\
81.0\end{array}$ & $\begin{array}{c}46.21 \\
(2)\end{array}$ & $<0.001$ \\
\hline & Total & $\begin{array}{c}\text { Agree } \\
\text { Neutral } \\
\text { Disagree }\end{array}$ & $\begin{array}{c}159 \\
7 \\
12\end{array}$ & $\begin{array}{l}47 \\
32 \\
27\end{array}$ & $\begin{array}{l}22.8 \\
82.1 \\
69.2 \\
\end{array}$ & $\begin{array}{c}68.86 \\
(2)\end{array}$ & $<0.001$ \\
\hline \multirow{3}{*}{$\begin{array}{l}\text { Emotionally } \\
\text { supported and } \\
\text { being helped } \\
\text { from family }\end{array}$} & $\begin{array}{l}\text { Normal } \\
\text { Vaginal } \\
\text { Delivery }\end{array}$ & $\begin{array}{c}\text { Agree } \\
\text { Neutral } \\
\text { Disagree }\end{array}$ & $\begin{array}{c}89 \\
4 \\
8\end{array}$ & $\begin{array}{c}23 \\
10 \\
8\end{array}$ & $\begin{array}{l}20.5 \\
71.4 \\
50.0\end{array}$ & $\begin{array}{c}19.61 \\
(2)\end{array}$ & $<0.001$ \\
\hline & $\begin{array}{l}\text { Cesarean } \\
\text { Section }\end{array}$ & $\begin{array}{c}\text { Agree } \\
\text { Neutral } \\
\text { Disagree }\end{array}$ & $\begin{array}{c}70 \\
3 \\
4 \\
\end{array}$ & $\begin{array}{l}32 \\
11 \\
22 \\
\end{array}$ & $\begin{array}{l}31.4 \\
78.6 \\
84.6 \\
\end{array}$ & $\begin{array}{l}30.39 \\
(2)\end{array}$ & $<0.001$ \\
\hline & Total & $\begin{array}{c}\text { Agree } \\
\text { Neutral } \\
\text { Disagree }\end{array}$ & $\begin{array}{c}159 \\
7 \\
12 \\
\end{array}$ & $\begin{array}{l}55 \\
21 \\
30\end{array}$ & $\begin{array}{l}25.7 \\
75.0 \\
71.4\end{array}$ & $\begin{array}{l}50.23 \\
(2)\end{array}$ & $<0.001$ \\
\hline \multirow{3}{*}{$\begin{array}{l}\text { Can consult } \\
\text { family in case of } \\
\text { any problem }\end{array}$} & $\begin{array}{l}\text { Normal } \\
\text { Vaginal } \\
\text { Delivery }\end{array}$ & $\begin{array}{c}\text { Agree } \\
\text { Neutral } \\
\text { Disagree }\end{array}$ & $\begin{array}{c}85 \\
6 \\
10\end{array}$ & $\begin{array}{c}25 \\
7 \\
9\end{array}$ & $\begin{array}{l}22.7 \\
53.8 \\
47.4\end{array}$ & $\begin{array}{c}9.14 \\
(2)\end{array}$ & 0.01 \\
\hline & $\begin{array}{l}\text { Cesarean } \\
\text { Section }\end{array}$ & $\begin{array}{c}\text { Agree } \\
\text { Neutral } \\
\text { Disagree }\end{array}$ & $\begin{array}{c}71 \\
4 \\
2\end{array}$ & $\begin{array}{c}41 \\
8 \\
16\end{array}$ & $\begin{array}{l}36.6 \\
66.7 \\
88.9\end{array}$ & $\begin{array}{c}19.38 \\
(2)\end{array}$ & $<0.001$ \\
\hline & Total & $\begin{array}{c}\text { Agree } \\
\text { Neutral } \\
\text { Disagree }\end{array}$ & $\begin{array}{c}156 \\
10 \\
12\end{array}$ & $\begin{array}{l}66 \\
15 \\
25\end{array}$ & $\begin{array}{l}29.7 \\
60.0 \\
67.6\end{array}$ & $\begin{array}{l}25.43 \\
(2)\end{array}$ & $<0.001$ \\
\hline $\begin{array}{l}\text { Family helps in } \\
\text { decision making } \\
\text { matters }\end{array}$ & $\begin{array}{l}\text { Normal } \\
\text { Vaginal } \\
\text { Delivery }\end{array}$ & $\begin{array}{c}\text { Agree } \\
\text { Neutral } \\
\text { Disagree }\end{array}$ & $\begin{array}{c}90 \\
7 \\
4\end{array}$ & $\begin{array}{c}22 \\
7 \\
12\end{array}$ & $\begin{array}{l}19.6 \\
50.0 \\
75.0\end{array}$ & $\begin{array}{c}24.26 \\
(2)\end{array}$ & $<0.001$ \\
\hline
\end{tabular}




\begin{tabular}{|c|c|c|c|c|c|c|c|}
\hline & $\begin{array}{l}\text { Cesarean } \\
\text { Section }\end{array}$ & $\begin{array}{c}\text { Agree } \\
\text { Neutral } \\
\text { Disagree }\end{array}$ & $\begin{array}{c}70 \\
3 \\
4\end{array}$ & $\begin{array}{c}30 \\
8 \\
27\end{array}$ & $\begin{array}{l}30.0 \\
72.7 \\
87.1\end{array}$ & $\begin{array}{c}34.57 \\
(2)\end{array}$ & $<0.001$ \\
\hline & Total & $\begin{array}{c}\text { Agree } \\
\text { Neutral } \\
\text { Disagree }\end{array}$ & $\begin{array}{c}160 \\
10 \\
8\end{array}$ & $\begin{array}{l}52 \\
15 \\
39 \\
\end{array}$ & $\begin{array}{l}24.5 \\
60.0 \\
83.0\end{array}$ & $\begin{array}{c}62.21 \\
(2)\end{array}$ & $<0.001$ \\
\hline \multicolumn{8}{|c|}{ Perceived Social Support from Friends } \\
\hline \multirow{3}{*}{$\begin{array}{c}\text { Have some } \\
\text { helping friends }\end{array}$} & $\begin{array}{l}\text { Normal } \\
\text { Vaginal } \\
\text { Delivery }\end{array}$ & $\begin{array}{c}\text { Agree } \\
\text { Neutral } \\
\text { Disagree }\end{array}$ & $\begin{array}{c}86 \\
6 \\
9\end{array}$ & $\begin{array}{l}20 \\
11 \\
10\end{array}$ & $\begin{array}{l}18.9 \\
64.7 \\
52.6\end{array}$ & $\begin{array}{c}21.02 \\
(2)\end{array}$ & $<0.001$ \\
\hline & $\begin{array}{l}\text { Cesarean } \\
\text { Section }\end{array}$ & $\begin{array}{c}\text { Agree } \\
\text { Neutral } \\
\text { Disagree }\end{array}$ & $\begin{array}{c}59 \\
12 \\
6\end{array}$ & $\begin{array}{l}28 \\
12 \\
25\end{array}$ & $\begin{array}{l}32.2 \\
50.0 \\
80.6\end{array}$ & $\begin{array}{c}21.83 \\
(2)\end{array}$ & $<0.001$ \\
\hline & Total & $\begin{array}{c}\text { Agree } \\
\text { Neutral } \\
\text { Disagree }\end{array}$ & $\begin{array}{c}145 \\
18 \\
15\end{array}$ & $\begin{array}{l}48 \\
23 \\
35\end{array}$ & $\begin{array}{l}24.9 \\
56.1 \\
70.0\end{array}$ & $\begin{array}{c}41.79 \\
(2)\end{array}$ & $<0.001$ \\
\hline \multirow{3}{*}{$\begin{array}{l}\text { Able to rely on } \\
\text { friends when } \\
\text { matters go } \\
\text { wrong }\end{array}$} & $\begin{array}{l}\text { Normal } \\
\text { Vaginal } \\
\text { Delivery }\end{array}$ & $\begin{array}{c}\text { Agree } \\
\text { Neutral } \\
\text { Disagree }\end{array}$ & $\begin{array}{l}77 \\
10 \\
14\end{array}$ & $\begin{array}{c}29 \\
4 \\
8\end{array}$ & $\begin{array}{l}27.4 \\
28.6 \\
36.4\end{array}$ & $\begin{array}{l}0.72 \\
(2)\end{array}$ & 0.69 \\
\hline & $\begin{array}{l}\text { Cesarean } \\
\text { Section }\end{array}$ & $\begin{array}{c}\text { Agree } \\
\text { Neutral } \\
\text { Disagree }\end{array}$ & $\begin{array}{c}52 \\
7 \\
18\end{array}$ & $\begin{array}{l}39 \\
11 \\
15\end{array}$ & $\begin{array}{l}42.9 \\
61.1 \\
45.5\end{array}$ & $\begin{array}{c}2.02 \\
(2)\end{array}$ & 0.36 \\
\hline & Total & $\begin{array}{c}\text { Agree } \\
\text { Neutral } \\
\text { Disagree }\end{array}$ & $\begin{array}{c}129 \\
17 \\
32\end{array}$ & $\begin{array}{l}68 \\
15 \\
23 \\
\end{array}$ & $\begin{array}{l}34.5 \\
46.9 \\
41.8\end{array}$ & $\begin{array}{l}2.38 \\
(2)\end{array}$ & 0.30 \\
\hline \multirow{3}{*}{$\begin{array}{l}\text { Have friends to } \\
\text { share worries } \\
\text { and happiness } \\
\text { with them }\end{array}$} & $\begin{array}{l}\text { Normal } \\
\text { Vaginal } \\
\text { Delivery }\end{array}$ & $\begin{array}{c}\text { Agree } \\
\text { Neutral } \\
\text { Disagree }\end{array}$ & $\begin{array}{c}81 \\
13 \\
7\end{array}$ & $\begin{array}{c}24 \\
13 \\
4\end{array}$ & $\begin{array}{l}22.9 \\
50.0 \\
36.4\end{array}$ & $\begin{array}{l}7.80 \\
(2)\end{array}$ & 0.02 \\
\hline & $\begin{array}{l}\text { Cesarean } \\
\text { Section }\end{array}$ & $\begin{array}{c}\text { Agree } \\
\text { Neutral } \\
\text { Disagree }\end{array}$ & $\begin{array}{c}57 \\
12 \\
8\end{array}$ & $\begin{array}{c}41 \\
4 \\
20\end{array}$ & $\begin{array}{l}41.8 \\
25.0 \\
71.4\end{array}$ & $\begin{array}{c}10.82 \\
(2)\end{array}$ & 0.004 \\
\hline & Total & $\begin{array}{c}\text { Agree } \\
\text { Neutral } \\
\text { Disagree }\end{array}$ & $\begin{array}{l}138 \\
25 \\
15\end{array}$ & $\begin{array}{l}65 \\
17 \\
24\end{array}$ & $\begin{array}{l}32.0 \\
40.5 \\
61.5\end{array}$ & $\begin{array}{c}12.39 \\
(2)\end{array}$ & 0.002 \\
\hline \multirow{3}{*}{$\begin{array}{l}\text { Can consult } \\
\text { friends in case } \\
\text { of any problem }\end{array}$} & $\begin{array}{l}\text { Normal } \\
\text { Vaginal } \\
\text { Delivery }\end{array}$ & $\begin{array}{c}\text { Agree } \\
\text { Neutral } \\
\text { Disagree }\end{array}$ & $\begin{array}{c}82 \\
7 \\
12\end{array}$ & $\begin{array}{c}22 \\
9 \\
10\end{array}$ & $\begin{array}{l}21.2 \\
56.2 \\
45.5\end{array}$ & $\begin{array}{c}11.80 \\
(2)\end{array}$ & 0.003 \\
\hline & $\begin{array}{c}\text { Cesarean } \\
\text { Section }\end{array}$ & $\begin{array}{c}\text { Agree } \\
\text { Neutral } \\
\text { Disagree }\end{array}$ & $\begin{array}{c}57 \\
6 \\
14\end{array}$ & $\begin{array}{c}36 \\
4 \\
25\end{array}$ & $\begin{array}{l}38.7 \\
40.0 \\
64.1\end{array}$ & $\begin{array}{l}7.28 \\
(2)\end{array}$ & 0.02 \\
\hline & Total & $\begin{array}{c}\text { Agree } \\
\text { Neutral } \\
\text { Disagree }\end{array}$ & $\begin{array}{c}139 \\
13 \\
26\end{array}$ & $\begin{array}{l}58 \\
13 \\
35\end{array}$ & $\begin{array}{l}29.4 \\
50.0 \\
57.4\end{array}$ & $\begin{array}{c}17.50 \\
(2)\end{array}$ & $<0.001$ \\
\hline
\end{tabular}

The Simple Linear Regression was done to measure association of Global perceived social support with postnatal depression among women in normal vaginal delivery, cesarean section and in total sample. The global perceived social support and postnatal depression were measured in terms of scores. A significant regression equation was found among women in normal vaginal delivery group $(F(1,140)=$ 5.02, $\mathrm{p}=0.02)$, with an $\mathrm{R}^{2}$ of 0.03 ; women in cesarean section group $(F(1,140)=21.41, p<0.001)$, with an $R^{2}$ of 0.13 ; and in total sample $(F(1,282)=$ 29.46, $p<0.001$ ), with an $R^{2}$ of 0.09 .
The global perceived social support was found as significant protective factor for postnatal depression among women in normal vaginal delivery group, in cesarean section group and in total sample. With onepoint increase in global perceived social support, the postnatal depression decreased by 0.1 points on EPDS scale $(p=0.03)$ among women in normal vaginal delivery group, by 0.2 points in cesarean section group $(p<0.001)$ and the postnatal depression decreased by 0.2 points in total sample ( $p$ $<0.001$ ) (Table 3). 
Table 3. Simple Linear Regression to Measure Association of Global Perceived Social Support with Postnatal Depression in Normal Vaginal Delivery Group $(n=142)$ and Cesarean Section Group $(n=142)$ Separately and Total $(n=284)$.

\begin{tabular}{|c|c|c|c|c|c|}
\hline \multirow{2}{*}{ Global Perceived Social Support } & \multicolumn{2}{|c|}{$\begin{array}{c}\text { Unstandardized } \\
\text { Coefficients }\end{array}$} & $\begin{array}{c}\text { Standardized } \\
\text { Coefficients }\end{array}$ & \multirow{2}{*}{ t } & \multirow{2}{*}{ p-value } \\
\cline { 2 - 6 } & B & S.E & Beta & & \\
\hline Normal Vaginal Delivery & -0.09 & 0.04 & -0.18 & -2.24 & 0.03 \\
\hline Cesarean Section & -0.17 & 0.04 & -0.36 & -4.63 & $<0.001$ \\
\hline Total & -0.15 & 0.03 & -0.31 & -5.42 & $<0.001$ \\
\hline
\end{tabular}

\section{DISCUSSION}

The current study revealed significant association of various perceived social support indicators with postnatal depression. The women who received social support from their family, friends and significant other were found as less depressed compared to those women who were deprived of social support. The 'perceived social support from significant other', 'perceived social support from family' and 'perceived social support from friends' were found as significant predictors of postnatal depression among mothers of both groups (who had C-section and vaginal delivery). The findings of a similar study showed that the women who had their husband's support for the child care and women who had contented marital relationship had lower risk of having postnatal depression[16]. Another study confirmed the significant association of social support with postnatal depression and suggested that the lack of home support was a contributory factor towards having postnatal depression among puerperal women[17].Gebregziabher et al found the lack of support from husband as a significant risk factor for developing postpartum depression [18]. The logistic regression analysis done by Ozmen et al measured a significant association of perceived social support from significant other and perceived social support from family with postnatal depression, however, no association was found between perceived social support from friends with postnatal depression [19]. Husain et al found perceived social support from significant other, from family as well as from friends as significant predictors of postnatal depression, the perceived support being measured by MSPSS and postnatal depression measured with EPDS [20].

Waqas et al measured social support by using Social Provision Scale (SPS) and measured depression with
Hospital Anxiety and Depression scale (HADS). Their study established the fact that decreased scores on SPS scale contributed to increased scores on HADS [21]. Shitu et al [22], Wubetu et al [23], Kakyo et al [24],Hamdan et a[25] and Eastwood et a[26] established a strong association between poor social support and increased maternal depressive symptoms during postpartum period. Ege et al also found a negative correlation between social support and postnatal depression [27].

Leonard et al in their study revealed that the perceived social support decreased with the passage of time from 1 to 18 months postpartum. At 6 months postpartum the lack of social support significantly contributed to postnatal depression [28].Azale et al demonstrated that the social support is a protective factor in the society against postnatal depression and could become a risk factor if society ignores the importance of psychosocial support for mother during the postpartum period [29].Salem et al found no significant association of perceived social support with postnatal depression [30].

\section{CONCLUSION}

A poor perceived social support from significant other, family and friends is a significant risk factor for postnatal depression among puerperal women.

\section{REFERENCES}

1. Morrell CJ, Sutcliffe P, Booth A, Stevens J, Scope $A$, Stevenson $M$, et al. A systematic review, evidence synthesis and meta-analysis of quantitative and qualitative studies evaluating the clinical effectiveness, the cost-effectiveness, safety and acceptability of interventions to prevent postnatal depression. Health Technology Assessment. 2016;20(37). 
2. Patel V, Rodrigues M, DeSouza N. Gender, poverty, and postnatal depression: a study of mothers in Goa, India. American journal of Psychiatry. 2002;159(1):43-7.

3. Rahman A, lqbal Z, Harrington R. Life events, social support and depression in childbirth: perspectives from a rural community in the developing world. Psychological medicine. 2003;33(7):1161-7.

4. Alasoom LI, Koura MR. Predictors of postpartum depression in the eastern province capital of Saudi Arabia. Journal of family medicine and primary care. 2014;3(2):146.

5. Veisani $Y$, Delpisheh A, Sayehmiri K, Rezaeian S. Trends of postpartum depression in iran: a systematic review and meta-analysis. Depression research and treatment. 2013;2013.

6. Shrestha N, Hazrah P, Sagar R. Incidence and prevalence of postpartum depression in a rural community of India. Journal of Chitwan Medical College. 2015;5(2):11-9.

7. Topatan S, Demirci N. Frequency of Depression and Risk Factors among Adolescent Mothers in Turkey within the First Year of the Postnatal Period. J Pediatr Adolesc Gynecol. 2019.

8. Lucci TK, Otta E. Postpartum depression and child development in first year of life. Estudos de Psicologia (Campinas). 2013;30(1):7-17.

9. Ghaedrahmati M, Kazemi A, Kheirabadi G, Ebrahimi A, Bahrami M. Postpartum depression risk factors: A narrative review. Journal of education and health promotion. 2017;6.

10. Roumieh M, Bashour H, Kharouf M, Chaikha S. Prevalence and risk factors for postpartum depression among women seen at Primary Health Care Centres in Damascus. BMC Pregnancy Childbirth. 2019;19(1):519.

11. Smorti M, Ponti L, Pancetti F. A Comprehensive Analysis of Post-partum Depression Risk Factors: The Role of Socio-Demographic, Individual, Relational, and Delivery Characteristics. Frontiers in Public Health. 2019;7(295).

12. Ghaedrahmati M, Kazemi A, Kheirabadi G, Ebrahimi A, Bahrami M. Postpartum depression risk factors: A narrative review. J Educ Health Promot. 2017;6:60-.

13. Mohammad Redzuan SA, Suntharalingam $P$, Palaniyappan T, Ganasan V, Megat Abu Bakar PN, Kaur P, et al. Prevalence and risk factors of postpartum depression, general depressive symptoms, anxiety and stress (PODSAS) among mothers during their 4-week postnatal follow-up in five public health clinics in Perak: A study protocol for a cross-sectional study. BMJ Open. 2020;10(6):e034458.

14. Wolf AW, De Andraca I, Lozoff B. Maternal depression in three Latin American samples. Social Psychiatry and Psychiatric Epidemiology. 2002;37(4):169-76.

15. Dolatian M, Maziar P, Majd HA, Yazdjerdi M. The relationship between mode of delivery and postpartum depression. Journal of Reproduction \& Infertility. 2006;7(3).

16. Mohamad Yusuff AS, Tang L, Binns CW, Lee AH. Prevalence and risk factors for postnatal depression in Sabah, Malaysia: a cohort study. Women Birth. 2015;28(1):25-9.

17. Alzahrani AD. Risk Factors for Postnatal Depression among Primipara Mothers. Span J Psychol. 2019;22:E35.

18. Gebregziabher NK, Netsereab TB, Fessaha YG, Alaza FA, Ghebrehiwet NK, Sium AH. Prevalence and associated factors of postpartum depression among postpartum mothers in central region, Eritrea: a health facility based survey. BMC Public Health. 2020;20(1):1614.

19. Ozmen D, Cetinkaya AC, Ulas SC, Ozmen E. Association between perceived social support and postpartum depression in Turkey. Journal of Advances in Medicine and Medical Research. 2014:2025-36.

20. Husain N, Bevc I, Husain M, Chaudhry I, Atif N, Rahman A. Prevalence and social correlates of postnatal depression in a low income country. Archives of women's mental health. 2006;9(4):197202.

21. Waqas A, Raza N, Lodhi HW, Muhammad Z, Jamal M, Rehman A. Psychosocial factors of antenatal anxiety and depression in Pakistan: is social support a mediator? PloS one. 2015;10(1):e0116510.

22. Shitu S, Geda B, Dheresa M. Postpartum depression and associated factors among mothers who gave birth in the last twelve months in Ankesha district, Awi zone, North West Ethiopia. BMC pregnancy and childbirth. 2019;19(1):435.

23. Wubetu AD, Engidaw NA, Gizachew KD. Prevalence of postpartum depression and associated factors among postnatal care attendees in Debre Berhan, Ethiopia, 2018. BMC Pregnancy and Childbirth. 2020;20:1-9.

24. Kakyo TA, Muliira JK, Mbalinda SN, Kizza IB, Muliira RS. Factors associated with depressive symptoms among postpartum mothers in a rural district in Uganda. Midwifery. 2012;28(3):374-9. 
25. Hamdan A, Tamim H. Psychosocial risk and protective factors for postpartum depression in the United Arab Emirates. Archives of women's mental health. 2011;14(2):125-33.

26. Eastwood JG, Jalaludin BB, Kemp LA, Phung HN, Barnett BEW. Relationship of postnatal depressive symptoms to infant temperament, maternal expectations, social support and other potential risk factors: findings from a large Australian crosssectional study. BMC pregnancy and childbirth. 2012;12(1):148.

27. Ege E, Timur S, Zincir H, Geçkil E, Sunar-Reeder B. Social support and symptoms of postpartum depression among new mothers in Eastern Turkey. The journal of obstetrics and gynaecology research. 2008;34(4):585-93.
28. Leonard KS, Evans MB, Kjerulff KH, Symons Downs D. Postpartum Perceived Stress Explains the Association between Perceived Social Support and Depressive Symptoms. Women's health issues : official publication of the Jacobs Institute of Women's Health. 2020;30(4):231-9.

29. Azale T, Fekadu A, Hanlon C. Postpartum depressive symptoms in the context of high social adversity and reproductive health threats: a population-based study. International journal of mental health systems. 2018;12(1):42.

30. Salem MN, Thabet MN, Fouly H, Abbas AM. Factors affecting the occurrence of postpartum depression among puerperal women in Sohag city, Egypt. Proceedings in Obstetrics and Gynecology. 2017;7(1):1-10.

This is an Open Access article distributed under the terms of the Creative Commons Attribution License (http://creativecommons.org/licenses/by/4.0), which permits unrestricted use, distribution, and reproduction in any medium, provided the original work is properly cited. 